\title{
The solitary waves of nonlinear Dirac equation in complex systems
}

\author{
Zhengning Gan ${ }^{1, a}$ \\ ${ }^{1}$ College of Electronic and Information Engineering, Changsha University of Science \& Technology, Changsha, \\ 410015, China
}

Keywords: solitary wave, nonlinear Dirac equation, external fields

\begin{abstract}
We adopt suitable numerical method to study the solitary waves of nonlinear Dirac equation in complex system. In some conditions, using nonlinear Dirac equation, we can better describe the macroscopic electrical phenomena and features generated by large amounts of electrons. As a result, we found the solitary wave's integral parameter plays an important role. With the increase of the integral parameter, the solitary wave's characteristic scale is becoming smaller, and the solitary wave more and more like a classical particle.
\end{abstract}

\section{Introduction}

In 1928 the British physicist P. Dirac presented a relativistic quantum mechanics equation which describes the movement of an electron, namely, Dirac equation [1]. It is the Lorentz covariant modified Schrödinger equation, and can meet the special theory of relativity and quantum mechanics simultaneously. However, Dirac equation, even taking into account the role of non-self-excitation field, can only be seen as the linear partial differential equations. The so-called linear equations satisfy the principle of linear superposition, and linear superposition of any two solutions is still a solution of the equation.

The scholars proposed more and more other nonlinear Dirac spinor field schemes [2-10], which can be applied in a mass of complex systems, including the nonlinear chemical reaction diffusion system, the complex biological tissue system, etc. In some conditions, using the nonlinear equation, we can more convenient to describe the macroscopic electrical phenomena and features generated by large amounts of electrons [5].

\section{Nonlinear Dirac model}

Using natural working unit system ( $c=\hbar=\mu_{0}=1$ ), the Lagrangian action density of Dirac equation under the electromagnetic fields is written by

$L_{D}=i \bar{\psi} \gamma^{\alpha} \partial_{\alpha} \psi-e \bar{\psi} \gamma^{\alpha} A_{\alpha} \psi-\mu \bar{\psi} \psi+f(\bar{\psi}, \psi)$,

a Zhengning Gan : gzn8060@163.com

A Project Supported by Scientific Research Fund of Hunan Provincial Education Department 
where $\psi$ is the spinor field, $\bar{\psi}=\psi^{+} \gamma^{0}$, and $\gamma^{\alpha}$ are Dirac matrices which meet the relations $\gamma^{\alpha} \gamma^{\beta}+\gamma^{\beta} \gamma^{\alpha}=2 \eta^{\alpha \beta}, \mu$ is a constant, and $f(\bar{\psi}, \psi)=\lambda(\bar{\psi} \psi)^{2}$ is a nonlinear selfenergy item, and ignore the effects excited by coupled electromagnetic field [11]. So the action of the nonlinear Dirac system is

$J=\int_{t_{0}}^{t_{1}} \int_{\Omega} L_{D} d^{3} x d t$

By means of the variational principle, we obtain the nonlinear Dirac field equation as

$$
i \gamma^{\alpha} \partial_{\alpha} \psi-\mu \psi=e \gamma^{\alpha} A_{\alpha} \psi+2 \lambda(\bar{\psi} \psi) \psi \text {. }
$$

It is clear that the constant $\lambda$ can determine the strength of nonlinear interaction.

\section{The steady state solutions of the solitary wave}

Now we consider the steady state solitary wave solutions. In this case, the Dirac feild $\psi(x)=\phi(\vec{X}) e^{-i E t}$, the external electromagnetic vector fields $A_{\mu}(x)=U_{\mu}(\vec{X})$, where $x=(t, \vec{X})$. Then we obtain the steady state equation as

$$
E \gamma^{0} \phi+i \gamma^{k} \partial_{k} \phi-\mu \phi=e \gamma^{\alpha} U_{\alpha} \phi+2 \lambda(\bar{\phi} \phi) \phi \text {. }
$$

This is a nonlinear eigenvalue equation, and the eigenfunction is $\phi$, the eigenvalue energy is $E$. The above equation can also be expressed by

$$
E=\left(-i \bar{\phi} \gamma^{k} \partial_{k} \phi+\mu \bar{\phi} \phi+e U_{\alpha} \bar{\phi} \gamma^{\alpha} \phi+2 \lambda(\bar{\phi} \phi)^{2}\right) /\left(\phi^{+} \phi\right) \text {. }
$$

Assuming that the characteristic space scale is $([-L, L],[-L, L],[-L, L])$, using the variational principle, we obtain the following solitary wave approximate normalized solution:

$$
\begin{aligned}
& \psi(x, y, z, t)= \\
& A\left(1+\cos \left(\frac{\pi x}{L}\right)\right)\left(1+\cos \left(\frac{\pi y}{L}\right)\right)\left(1+\cos \left(\frac{\pi z}{L}\right)\right) e^{-i E_{0} t}, \\
& (x, y, z) \in([-L, L],[-L, L],[-L, L]) \\
& \psi(x, y, z, t)=0, \quad(x, y, z) \notin([-L, L],[-L, L],[-L, L])
\end{aligned}
$$

where the characteristic amplitude $A=\left[0.141956 \sqrt{\frac{\Lambda}{\lambda}}, 0,0,0\right]^{T}$, the characteristic length $L=1.224923\left(\frac{\lambda}{\Lambda}\right)^{1 / 3}$, the eignvalue $E_{0}=\mu-\Lambda>0$ and the integral parameter $\Lambda>0$. It is proved that the solitary wave is stable for small perturbations [1].

When the center point of the solitary wave is at the coordinate origin $(0,0,0)$, the wave function density distribution on the plane $Z=0$ is shown in Fig. 1. As the integral parameter increased from 0.1 to 0.7 , the peak of the wave function density increased from 0.12896 to 0.90279 , and the characteristic scale $L$ decreased from 2. 63902 down to 1.37957 . So we found the integral parameter $\Lambda$ plays an important role in forming the structure of the solitary wave.

\section{The motion of the solitary waves interacting with external fields}

Using (6) and (7), combined with Lorentz transformation, we can obtain a solitary wave solutions with linear motion: 


$$
\begin{aligned}
& \left.\psi(x, y, z, t)=A\left(1+\cos \left(\frac{\pi(x-X(t))}{L}\right)\right)\left(+\cos \frac{\pi(y-Y(t))}{L}\right)\right)\left(+\cos \frac{\pi(z-Z(t))}{L}\right) e^{i\left(p_{x} x+p_{y} y+p_{z} z-E t\right)}, \\
& (x, y, z) \in([-L, L],[-L, L],[-L, L]) \\
& \psi(x, y, z, t)=0, \quad(x, y, z) \notin([-L, L],[-L, L],[-L, L])
\end{aligned}
$$

where the amplitude parameter is given by

$$
A=0.141956\left(1+\left(p_{x}^{2}+p_{y}^{2}+p_{z}^{2}\right) /\left(E+E_{0}\right)\right)^{-1 / 2} \sqrt{\frac{\Lambda}{\lambda}}\left[1,0, \frac{p_{z}}{E+E_{0}}, \frac{p_{x}+i p_{y}}{E+E_{0}}\right]^{T}
$$

the group speeds are $V_{x}=p_{x} / E, V_{x}=p_{x} / E$ and $V_{x}=p_{x} / E$, and the energy $E=\sqrt{p_{x}^{2}+p_{y}^{2}+p_{Z}^{2}+E_{0}^{2}}$. The peak position $(X(t), Y(t), Z(t)) \quad$ is also the center position of the solitary wave, which is given by $(X(t), Y(t), Z(t))=\left(v_{x} t, v_{y} t, v_{z} t\right)$.

In the following, we will discuss the motion of the solitary waves interacting with external fields. Since the solitary wave is stable for small perturbations, we could assume that the shape of the solitary wave is approximately unchanged. So we could focus on the movement of the wave peak point of the solitary wave. Using the variational principle, we have

$$
\delta J=\delta \int_{t_{0}}^{t_{1}} \int_{\Omega} L_{D} d^{3} x d t=0,
$$

where $L_{D}$ is given by $L_{D}=i \bar{\psi} \gamma^{\alpha} \partial_{\alpha} \psi-e \bar{\psi} \gamma^{\alpha} A_{\alpha} \psi-\mu \bar{\psi} \psi+\lambda(\bar{\psi} \psi)^{2}$. Substituting (8) and (9) into (10), we obtain

$$
\delta \int_{t_{0}}^{t_{1}} \int_{\Omega}\left(-e \bar{\psi} \gamma^{\alpha} A_{\alpha} \psi-\lambda(\bar{\psi} \psi)^{2}\right) d^{3} x d t=0 .
$$

In the the frame of reference $\Sigma^{\prime}$, the origin point of which is always the center point of the solitary wave, we obtain

$$
\delta \int_{t_{0}^{\prime}}^{t_{1}^{\prime}} \int_{\Omega^{\prime}}\left(-e \bar{\psi}^{\prime} \gamma^{\alpha^{\prime}} A_{\alpha}^{\prime} \psi^{\prime}-\lambda\left(\bar{\psi}^{\prime} \psi^{\prime}\right)^{2}\right) d^{3} x^{\prime} d t^{\prime}=0
$$

For low speed conditions, applying the time dilation formula for fixed point measurement $d t^{\prime}=\sqrt{1-\vec{V}^{2}} d t$ (the peak velocity $\vec{V}=d \vec{X} / d t$ ), we have

$$
\begin{aligned}
& \psi^{\prime} \approx \psi^{\prime}\left(\vec{X}^{\prime}-\vec{X}^{\prime}(t), t\right), \\
& J^{0^{\prime}} A_{0}^{\prime} \approx J^{0^{\prime}} U^{\prime} \approx J^{0^{\prime}}\left(\vec{X}^{\prime}-\vec{X}^{\prime}(t)\right) U^{\prime}\left(\vec{X}^{\prime}-\vec{X}^{\prime}(t)+\vec{X}^{\prime}(t)\right),
\end{aligned}
$$

where $U^{\prime}\left(\vec{X}^{\prime}\right)$ is the external potential field. Substituting the above items into (12), we obtain

$$
\delta \int_{t_{0}}^{t_{1}}\left(-M \sqrt{1-\vec{V}^{2}}-e U(\vec{X})-\frac{1}{2} K_{0} \nabla^{2} U(\vec{X})\right) d t \approx 0 .
$$

Further simplification, we obtain

$$
\delta \int_{t_{0}}^{t_{1}}\left(-M+\frac{1}{2} M \vec{V}^{2}-e U(\vec{X})-\frac{1}{2} K_{0} \nabla^{2} U(\vec{X})\right) d t \approx 0,
$$

where the wave center position $\vec{X}=\vec{X}(t)$, the wave center velocity $\vec{V}=\vec{V}(t)=d \vec{X} / d t$, the solitary wave inertia quality $M=\Lambda / 2$, and the modified parameter $K_{0}=0.0080053(\lambda / \Lambda)^{2 / 3}$. Using the variational principle, we obtain the motion equation of the wave center:

$$
M \frac{d \vec{V}}{d t} \approx e \nabla U(\vec{X})-\frac{1}{2} K_{0} \nabla\left(\nabla^{2} U(\vec{X})\right) .
$$


Compared with the motion equation of classical particle, the additional item is $\frac{1}{2} K_{0} \nabla\left(\nabla^{2} U(\vec{X})\right)$.

We then study the trajectory of the center point of the solitary wave under the three-dimensional external potential $U=\frac{1}{2} M \omega^{2}\left(x^{2}+y^{2}+z^{2}\right)$. Since $\frac{1}{2} K_{0} \nabla\left(\nabla^{2} U(\vec{X})\right)=0$, the trajectory is as same as that of the particle in classical mechanics. Suppose the external potential field $U=\frac{1}{4} K_{1}\left(x^{2}+y^{2}+z^{2}\right)^{2}$, then $\frac{1}{2} K_{0} \nabla\left(\nabla^{2} U(\vec{X})\right) \neq 0$, which leads to the differences of the trajectories between the center point of the solitary wave and that of the classical particle, as shown in Figure 2. When the integral promoter $\Lambda=0.3$, We can observe that the quasimovement cycle is roughly 2.5 , and the amplitude increased from 0.04 to 0.048 . when $\Lambda=0.7$, the quasi-movement cycle is roughly 5.13 , and the amplitude increased slowly from 0.082 to 0.084 . When the integral parameters further increased to 0.9 , the movement cycle becomes 6. 28 . The right diagram in Fig. 2 describes the situation of the classical particle, and the cycle is near to 11.16 , the amplitude is 0.2 .

Since the characteristic amplitude $A_{0}=\left[0.141956 \sqrt{\frac{\Lambda}{\lambda}}, 0,0,0\right]^{T}$, the characteristic length $L=1.224923\left(\frac{\lambda}{\Lambda}\right)^{1 / 3}$, and the modified parameter $K_{0}=0.0080053(\lambda / \Lambda)^{2 / 3}$, we obtain $A_{0}^{2} \sim \Lambda, L \sim \Lambda^{-1 / 3}$, and $K_{0} \sim \Lambda^{-2 / 3}$. Overall, with the increase of the integral parameter $\Lambda$, the characteristic parameters of the solitary wave motion are constantly close to the relevant parameters of the classical particle.
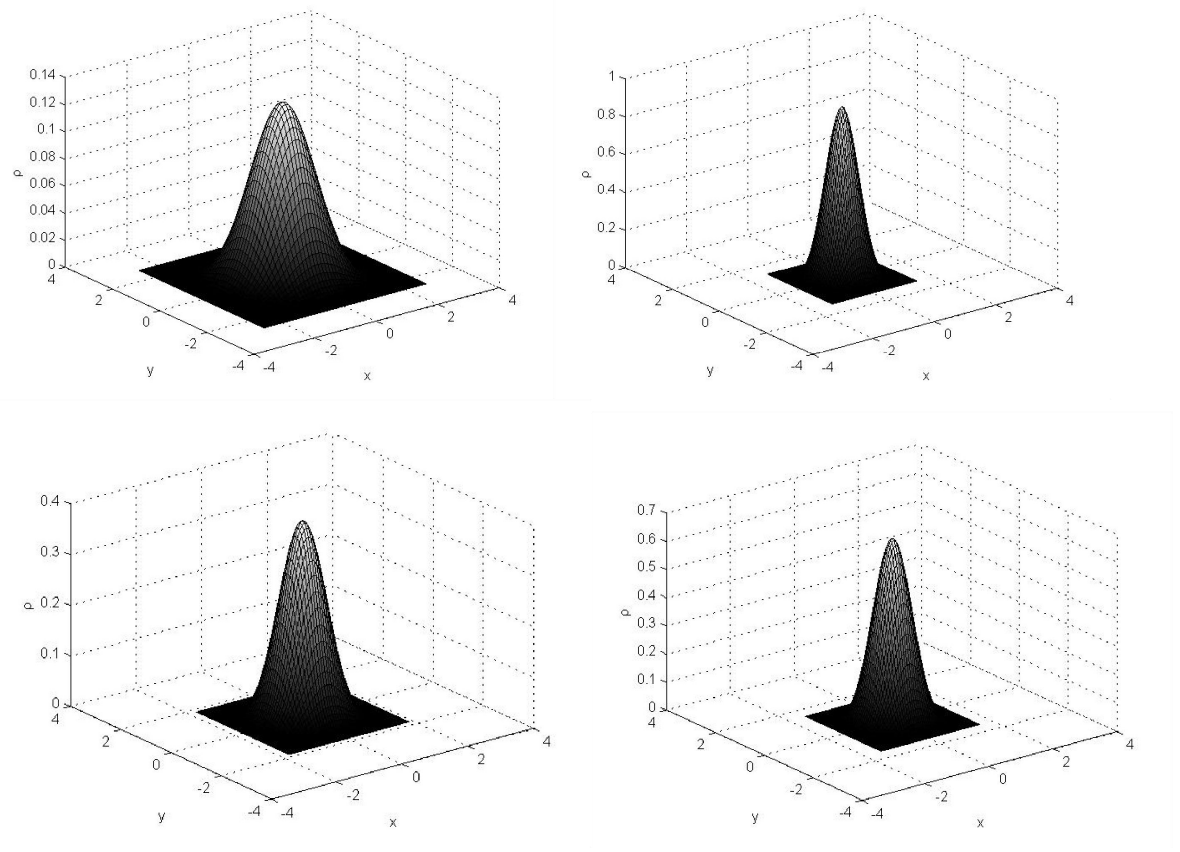

Figure 1.The wave function density distribution $\rho=\psi^{+} \psi$ on the plane $z=0$ is shown. From left to right and from up

to down, the integral parameter $\Lambda$ is selected as $0.1,0.3,0.5,0.7$, respectively. The parameters of the equation are given by $\mathrm{e}=1, \mu=1$ and $\lambda=1$. 

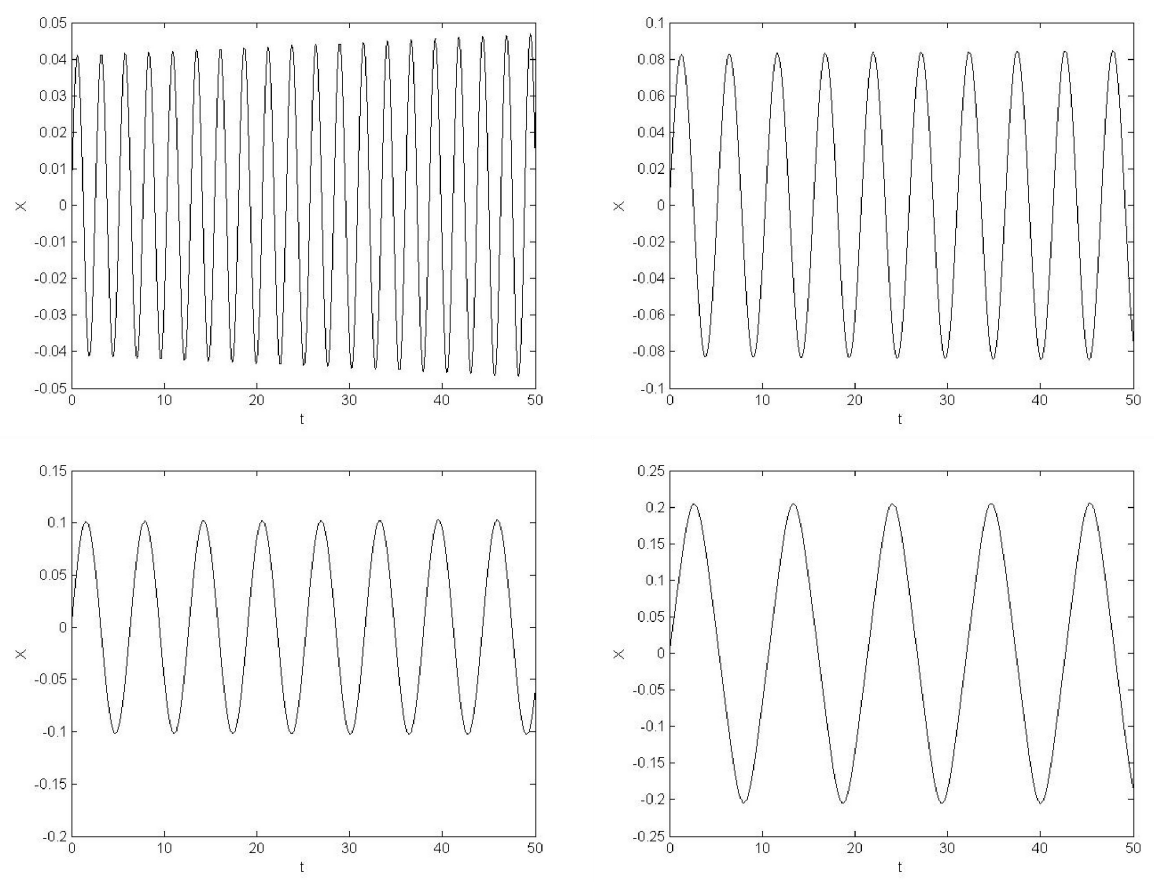

Figure 2.The evolution of the $\mathrm{X}$-axis coordinate of the center point. From left to right and from up to down, the integral parameter $\Lambda$ is selected as $0.3,0.7,0.9, \infty$, respectively. For classical particles, $\Lambda \rightarrow \infty$. The parameters of the equation are given by $\mathrm{e}=1, \mu=1$ and $\lambda=1$.

\section{Conclusions}

In summary, the theoretical analysis and numerical calculations show that the integral parameter plays a decisive role on the characteristics of the solitary wave. First, with the increase of the integral parameter, the characteristic scale is shrinking, and the characteristic magnitude is increasing. Secondly, the motion of the center point of the solitary wave is related with the integral parameter. With the increase of the integral parameter, the solitary wave more and more like a classical particle.

We are grateful to Professor Xinming Cheng for helpful proposal and discussion.

\section{References}

1. F.P. Miller, A.F. Vandome, J. McBrewster, Dirac Equation (VDM Publishing House Ltd. , 2009)

2. W. Fushchych, R. Zhdanov, Symmetries and Exact Solutions of Nonlinear Dirac Equations (Mathematical Ukraina Publisher, 1997)

3. A. Picozzi, J. Garnier, Media. Phys. Rev. Lett. 107, 233901 (2011)

4. J. Garaud, J. Carlström, E. Babaev, Phys. Rev. Lett. 107,197001 (2011)

5. Z.H. Wang, A. Hagerstrom, J.Q. Anderson, W. Tong, W.Z. Wu, L.D. Carr, R. Eykholt, B.A. Kalinikos, Phys. Rev. Lett. 107,114102 (2011)

6. S.V. Demidov, D.G. Levkov, Phys. Rev. Lett. 107 (2011), 071601.

7. J. Rajchenbach, A. Leroux, D. Clamond, Phys. Rev. Lett. 107, 024502. (2011)

8. S.P. Gorza, B. Deconinck, P. Emplit, T. Trogdon, M. Haelterman, Phys. Rev. Lett. 106, 094101. (2011)

9. W.H. Hai, Y. Xiao, Physics Letters A 208 (1995), 79-83.

10. P. Ghaemi, S. Ryu, Phys. Rev. B 85, 075111 (2012). 